\title{
Local oxidation nanolithography on Hf thin films using atomic force microscopy (AFM)
}

\author{
S Buyukkose, S Okur and G Aygun \\ Department of Physics, Izmir Institute of Technology, Izmir, TR 35430, Turkey \\ Received 8 February 2009 \\ Published 24 April 2009 \\ Online at stacks.iop.org/JPhysD/42/105302
}

\begin{abstract}
Well controlled Hf oxide patterns have been grown on a flat Hf thin film surface produced by the dc magnetron sputtering method on $\mathrm{Si}$ and $\mathrm{SiO}_{x}$ substrates. These patterns have been created by using the technique of semi-contact scanning probe lithography (SC-SPL). The thickness and width of the oxide patterns have been measured as a function of applied voltage, duration and relative humidity. There is a threshold voltage even at $87 \%$ humidity, due to insufficient energy required to start the oxide growth process for a measurable oxide protrusion. Electrical characterization was also performed via the $I-V$ curves of $\mathrm{Hf}$ and $\mathrm{HfO}_{x}$ structures, and the resistivity of $\mathrm{HfO}_{x}$ was found to be $4.284 \times 10^{9} \Omega \mathrm{cm}$. In addition to the $I-V$ curves, electric force microscopy and spreading surface resistance images of $\mathrm{Hf}$ and $\mathrm{HfO}_{x}$ were obtained.
\end{abstract}

(Some figures in this article are in colour only in the electronic version)

\section{Introduction}

Current microelectronics is based on silicon technology. However, silicon oxide has limitations in terms of electrical properties because of the actual physical scaling of microelectronic devices. The leakage current due to direct tunnelling degrades the circuit performance significantly, especially as the thickness of the silicon oxide layer becomes less than $3 \mathrm{~nm}$ [1]. An alternative is to replace the common dielectric material with higher dielectric $(\kappa)$ materials, such as $\mathrm{ZrO}_{2}, \mathrm{HfO}_{2}, \mathrm{Ta}_{2} \mathrm{O}_{5}, \mathrm{Y}_{2} \mathrm{O}_{3}$ and $\mathrm{TiO}_{2}$ [2,3]. It has been shown that $\mathrm{HfO}_{2}$, when grown on $\mathrm{Si}$, has excellent properties which include a high dielectric constant $(\kappa>20)$, low leakage current, good thermal and chemical stability with negligible dispersion and excellent reliability [4-6].

Patterning and fabrication of electronic devices at the nanometre scale are challenging. Common photolithography techniques are limited in resolution due to the interference effects inherent to the wave nature of UV light [7]. Therefore, SPM has recently received considerable attention as a promising tool for creating sub-100 $\mathrm{nm}$ structures for various applications such as single electron devices [8].

There are several $\mathrm{HfO}_{2}$ thin film growth techniques, such as electron-beam evaporation [9], $\mathrm{Hf}$ reactive sputtering in $\mathrm{O}_{2}$ atmosphere [4], laser oxidation [10,11] and scanning probe lithography (SPL) [12]. Among them, SPL allows nanoscale manipulations with high spatial resolution thus creating a controlled oxide pattern based on the electrochemical process between a conductive tip and the metallic surface under a bias voltage [13]. SPL is sometimes called local oxidation nanolithography (LON), scanning probe oxidation (SPO), nano-oxidation, local anodic oxidation (LAO) or AFM lithography [14]. It also has surface characterization advantages. Lo et al applied the anodic oxidation SPL method at relatively high voltages $(20-50 \mathrm{~V})$ on a hafnium oxide surface for silicon substrates [12].

In this work, semi-contact scanning probe lithography (SC-SPL) has been used to create hafnium oxide $\left(\mathrm{HfO}_{x}\right)$ patterns on bare hafnium (Hf) thin films under voltages less than $10 \mathrm{~V}$. There are several effective operating parameters such as strength of electric field, relative humidity, sample temperature and tip-surface distance. This study, however, focuses only on applied voltage (electric field strength), voltage duration and relative humidity. For SC-SPL operation, a conductive tip is kept at a negative potential with respect to the sample surface. The applied voltage creates an electric field with a strength of $\sim 10^{9} \mathrm{~V} \mathrm{~m}^{-1}$ between the tip and the sample surface. That strength has sufficient energy to decompose 


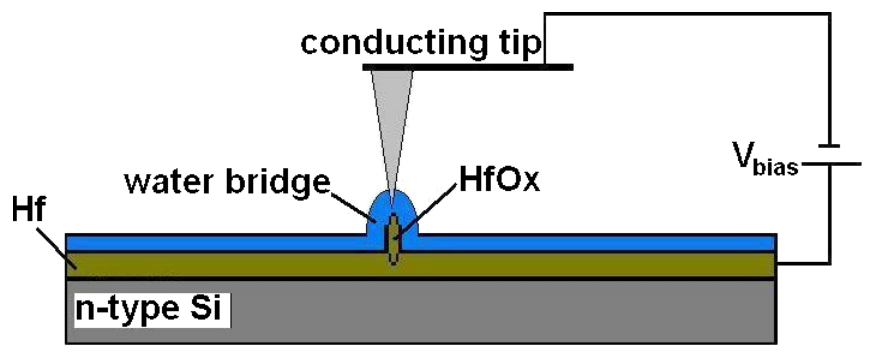

Figure 1. Representation of oxidation mechanism.

water molecules into hydrogen $\left(\mathrm{H}^{+}\right)$and hydroxide $\left(\mathrm{OH}^{-}\right)$ ions between the tip and the Hf surface. The negatively charged hydroxide ions are accelerated by the field to the surface of the hafnium film establishing the electrochemical oxidation process on the Hf film. The oxidation time, the magnitude of bias voltage and the relative humidity control the thickness and the protrusion width of the $\mathrm{HfO}_{x}$ nanopatterns.

A schematic diagram of SPM LAO of Hf metallic layer on an $\mathrm{Si}$ substrate is shown in figure 1. An adsorbed thin water layer, present at the surface, provides the required electrolyte for the electrochemical process under ambient conditions. The thickness of the water layer on the Hf surface can be controlled by relative humidity inside the SPM environmental chamber during the anodic oxidation process. When a negatively charged SPM tip is at a distance of approximately $10 \mathrm{~nm}$ from the Hf surface, decomposition of the water layer occurs due to the intense electric field $\left(\sim 1 \mathrm{~V} \mathrm{~nm}^{-1}\right)$ produced between the conductive SPM tip and the Hf surface. One of the possible tip-induced electrochemical reactions on the $\mathrm{Hf}$ surface can be described as according to the following chemical equation:

$$
\mathrm{H} f^{+}+2 x \mathrm{OH}^{-} \longrightarrow \mathrm{HfO}_{2 x}+x \mathrm{H}_{2} \text {. }
$$

\section{Experiment}

Hf thin films with $65 \mathrm{~nm}$ thickness were deposited on n-type $\mathrm{Si}\left(\begin{array}{lll}1 & 0 & 0\end{array}\right)$ by using the dc magnetron sputtering technique. For electrical characterization the Hf thin film was also deposited on a thermally oxidized $\mathrm{SiO}_{x}(\sim 336 \mathrm{~nm})$ layer on a Si wafer. During deposition, sputtering power was $30 \mathrm{~W}$ and base pressure was maintained at $2.2 \times 10^{-6}$ Torr. The substrate was located at a distance of $7.4 \mathrm{~cm}$ from the Hf target and was kept at $200{ }^{\circ} \mathrm{C}$. Prior to the SPL oxidation process, the surface roughness (root-mean-square value) of the sputtered Hf metallic surface was measured to be approximately $0.125 \mathrm{~nm}$ over an area of $25 \mu \mathrm{m}^{2}$. SPL anodic oxidation experiments were carried out by a commercial SPM (Solver P7, NT-MDT, Russia). All SPL oxidation processes were performed under ambient conditions at $24^{\circ} \mathrm{C}$ under four different relative humidities: $54 \%, 64 \%, 75 \%$ and $87 \%$. During the process, a positive bias was applied to the sample while the tip was grounded. A triangular diamond like carbon (DLC) coated conductive tip (made by NT-MDT) with a typical force constant of $48 \mathrm{~N} \mathrm{~m}^{-1}$ and apex radius of $75 \mathrm{~nm}$ was used
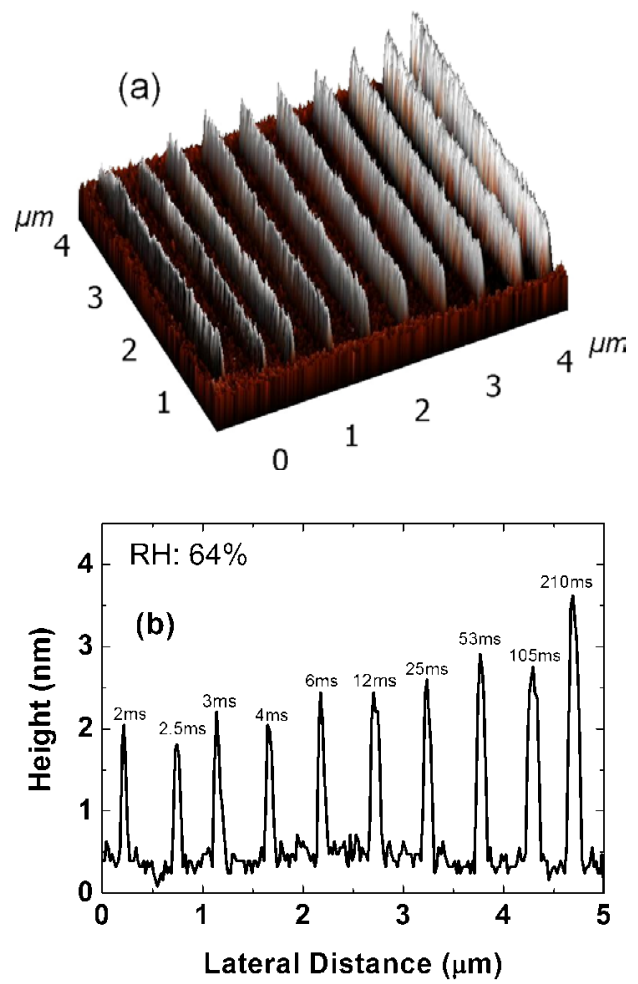

Figure 2. (a) 3D AFM image and (b) size profile of hafnium oxide patterns at various bias voltage durations ranging from 2 to $210 \mathrm{~ms}$. (Bias voltage: $10 \mathrm{~V}$, relative humidity: $64 \%$, temperature: $24^{\circ} \mathrm{C}$.)

during the oxidation and imaging processes of the grown oxide. AFM images were obtained in semi-contact mode at a resonance frequency of $360 \mathrm{kHz}$. The protrusion width of the oxide lines was obtained by measuring the full width at half maximum (FWHM).

\section{Results and discussion}

SPM based nano-oxidation lithography has been carried out by applying a continuous bias voltage on the surface of the sample while keeping the tip-surface spacing at a constant distance of $\sim 10 \mathrm{~nm}$ via the controlled feedback loop system. SC-SPL was chosen to obtain better control over oxide profiles in terms of oxide pattern uniformity and continuity $[15,16]$.

Figure 2( $a$ ) shows a 3D AFM image of the oxide lines fabricated under the same applied voltage to investigate the role of voltage time during the oxidation process. It shows that uniform oxide features are created under a relative humidity of $64 \%$ and $24^{\circ} \mathrm{C}$ substrate temperature. The oxide profile given in figure $2(b)$ reveals that there is a slight variation in thickness (around $1.5 \mathrm{~nm}$ ) as a function of bias voltage duration between 1 and $210 \mathrm{~ms}$. It is understood from this slight variation that most of the oxide formation takes place at the beginning of the process.

Several phenomenological models, such as the empirical power law $[17,18]$, the logarithmic function of the applied voltage duration [19] and the inverse exponential growth (IEG) function $[20,21]$ have been reported to predict the oxide thickness $(h)$ as a function of time $(t)$ and bias voltage $(V)$ 


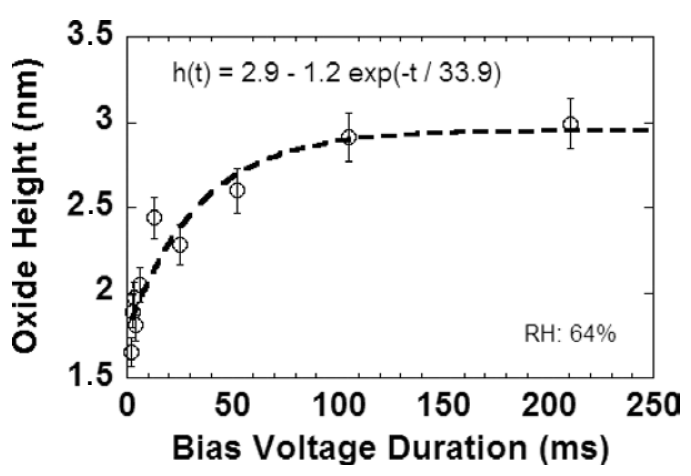

Figure 3. Oxide thickness versus applied bias voltage duration.

using the potential distribution in the oxide layer. This study shows that SPL grown $\mathrm{HfO}_{x}$ data were best fitted to the IEG model, which defines the oxide thickness with the following equations:

$$
\begin{aligned}
& \frac{\mathrm{d} h}{\mathrm{~d} t}=\left(\frac{h_{0}}{\tau}\right) \mathrm{e}^{-t / \tau}, \\
& h=h_{\mathrm{m}}-h_{0} \mathrm{e}^{-t / \tau},
\end{aligned}
$$

where $h$ and $t$ represent the oxide thickness and the pulse duration for the applied voltage, respectively. $h_{\mathrm{m}}$ and $h_{0}$ are the initial constants while $\tau$ is the time constant from the curve fit.

The best fit parameters for the IEG function were obtained as $h_{\mathrm{m}}=2.9 \mathrm{~nm}, h_{0}=1.2 \mathrm{~nm}$ and $\tau=33.9 \mathrm{~ms}$, as shown in figure 3. The oxidation process is faster at the beginning (up to $33.9 \mathrm{~ms}$ ), because there is only a water layer on the surface as a dielectric barrier. The oxidation rate reaches a plateau around $h_{\mathrm{m}}=2.9 \mathrm{~nm}$ due to $\mathrm{HfO}_{x}$ growth underneath the tip. Since the molecular volume of the Hf oxide layer is larger than that of Hf itself, the expansion of oxide volume causes stress along the growth direction in a limited local space at the $\mathrm{Hf} / \mathrm{HfO}_{x}$ interface, thus pushing the $\mathrm{HfO}_{x}$ layer upwards to form a protrusion. The higher oxide protrusion lowers the electric field between the tip and the surface, resulting in a decrease in the oxide growth rate due to an accumulation of dielectric material and ionic space charges trapped near the substrate/oxide interface $[17,18]$.

Figure 4(a) shows the two-dimensional AFM surface topography after anodic nano-oxidation lithography, and figure $4(b)$ demonstrates the profile of the created oxide lines with respect to applied bias voltages up to $10 \mathrm{~V}$ at $75 \%$ relative humidity. It can be noted that the oxide patterns have uniform features and display a distinct dependence on the applied bias voltage. The threshold voltage initiating Hf oxide growth for permanent measurable oxide line protrusions is around $6 \mathrm{~V}$ at room temperature and $75 \%$ relative humidity. The voltage dependence indicates that the electric field under the tip apex plays an important role in the local oxidation process. Higher bias voltages produce more ions and the kinetic energy of these ions is higher than that of ions produced under a lower electric field. The penetration depth of ions into the oxide layer depends on their energy. This means that more ions reach the $\mathrm{HfO}_{x} / \mathrm{Hf}$ interface under higher bias voltage. In this way, the oxidized region in the film increases with increasing bias voltage and thus a higher protrusion from the surface
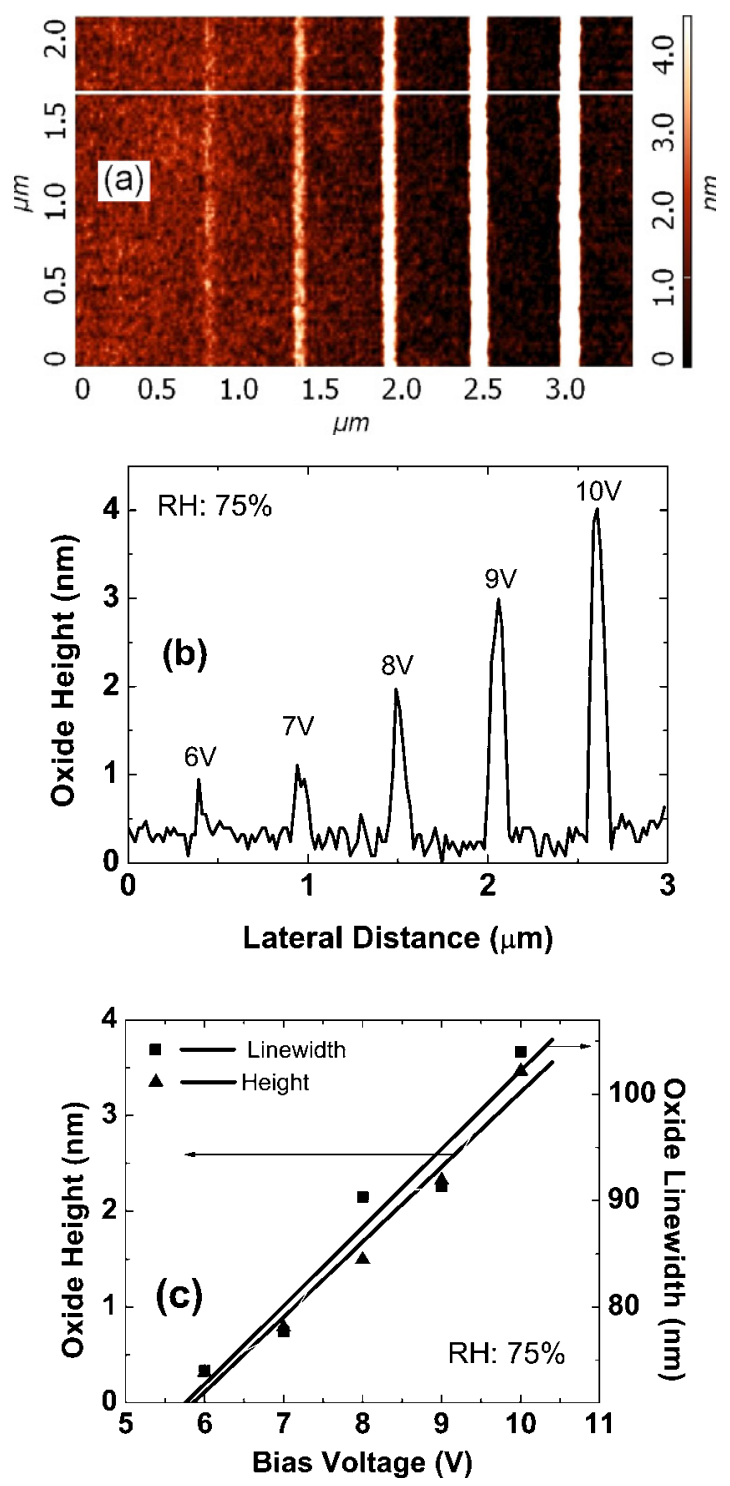

Figure 4. (a) 2D AFM image of oxide lines, (b) oxide thickness profiles obtained with various bias voltages from 6 to $10 \mathrm{~V}$ and (c) the thickness and the width of the oxide lines as a function of the applied bias voltage for $75 \%$ relative humidity.

is observed. Figure $4(c)$ shows the plot of oxide thickness $(h)$ versus applied voltage $(V)$ for oxides produced at $75 \%$ relative humidity and at the longest voltage duration $(210 \mathrm{~ms}$ for this study). It is clearly seen from figure $4(c)$ that there is a linear increase for both the oxide thickness and the oxide protrusion width with respect to applied bias voltage. Similar linear relationships have already been reported for $\mathrm{Si}$ and some other metals by various groups [20,22,23]. The electric field between the conductive AFM tip and the Hf metal surface is directly related to the applied voltage and the dielectric constant of the protruding oxide. The electric field causes $\mathrm{OH}^{-}$ions to easily diffuse through the oxide to the $\mathrm{Hf} / \mathrm{HfO}_{X}$ interface. The increase in the amount of oxide layer also causes the oxide protrusion width to increase.

Relative humidity is another effective parameter being the source of water layer on the Hf film. To understand the influence of the relative humidity on $\mathrm{HfO}_{x}$ thickness and 

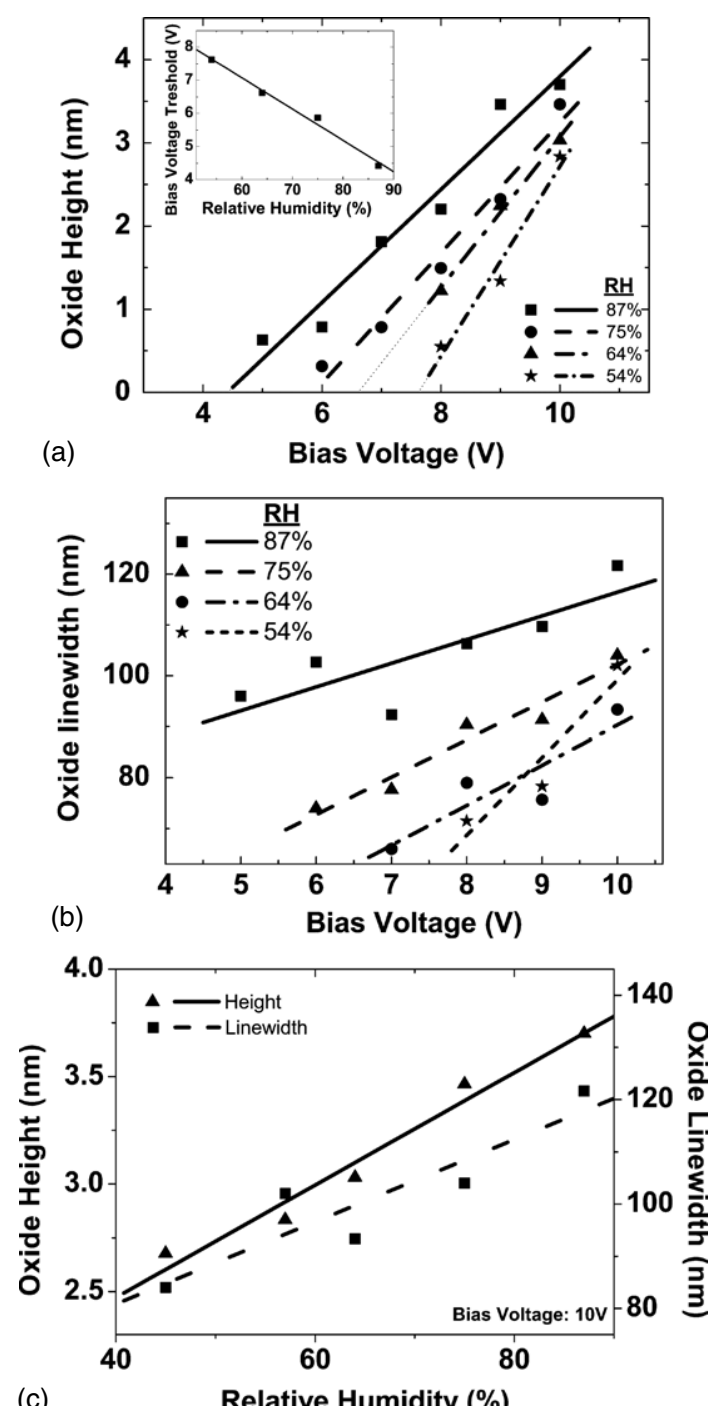

Figure 5. (a) Oxide thickness at various relative humidities. Tip bias voltage threshold value decreases linearly with increasing relative humidity (inset). (b) Oxide protrusion width versus applied bias voltage. $(c)$ The oxide thickness and the width with respect to the relative humidity under $10 \mathrm{~V}$ applied voltage.

width, SPL experiments were performed at humidities of $54 \%$, $64 \%, 75 \%$ and $87 \%$. Increasing humidity accelerates the oxide growth, as shown in figure $5(a)$. Both the thickness and the width of the resulting oxide lines depend on the amount of relative humidity due to the existing water film (figures $5(a)$ and $(b)$ ). Extrapolating the data, it can be concluded that the oxidation process starts when the sample bias voltage values are higher than $4.4 \mathrm{~V}, 5.9 \mathrm{~V}, 6.6 \mathrm{~V}$ and $7.6 \mathrm{~V}$ for relative humidities of $87 \%, 75 \%, 64 \%, 54 \%$, respectively. It is clearly seen that the threshold voltage for oxide formation decreases with increasing relative humidity (inset of figure 5(a)). The threshold voltage, oxide thickness and protrusion width dependence on $\mathrm{HfO}_{x}$ structures can be explained by taking account the number of water molecules between the tip and the sample surface. At low relative humidity there are not enough water molecules to provide the oxyanions for the oxidation reaction to proceed. Therefore, the threshold voltage starting the oxidation reaction will decrease with increasing relative humidity. During the oxidation process, the AFM feedback system was also activated to keep the distance between the tip and the surface constant; therefore, when the oxide layer starts to protrude, the AFM tip moves upwards in order to keep the distance constant. This makes the water bridge narrower, decreasing the number of ions, and the bridge will also be destroyed in a shorter time at low relative humidity. However, in the case of higher relative humidity, the number of created ions in the water bridge will increase and the water bridge will also exist for a longer time. The increase in relative humidity also result in a large contact area between the tip and the water, resulting in the formation of a larger bridge. Because the movement of ions is restricted by the boundary of water, ions can be spread across the surface to increase the protrusion width of oxide as a function of increasing relative humidity as shown in figure $5(c)$.

To investigate the electrical characteristics of $\mathrm{HfO}_{x}$ structures, an oxide pattern having a surface area of $1.42 \mu \mathrm{m} \times$ $1.26 \mu \mathrm{m}$ and a thickness of $3.3 \mathrm{~nm}$ was formed on an $\mathrm{Hf}$ thin film which had been deposited on a thermally oxidized $\mathrm{SiO}_{x}$ $(\sim 336 \mathrm{~nm})$ layer. The 3D surface topography and the thickness profile of this pattern can be seen in figures $6(a)$ and $(b)$. To obtain the current voltage characteristics of the protrusion of $\mathrm{HfO}_{x}$ and $\mathrm{Hf}$ film, the DLC coated conductive AFM tip was allowed to touch the $\mathrm{HfO}_{x}$ or $\mathrm{Hf}$ surface in contact mode as shown in figure $6(a)$ and two terminal electrical measurements were performed [24]. The distance between the conductive tip and the counter electrode was kept at $5 \mathrm{~mm}$. From the $I-V$ curves for the $\mathrm{HfO}_{x}$ and the $\mathrm{Hf}$ films, resistances of $8 \times 10^{12}$ and $40 \times 10^{6} \Omega$ were obtained, respectively, within their linear regions (figure $6(c)$ ). The corresponding resistivity of $\mathrm{HfO}_{x}$ was calculated from $\rho=R A / L$, where $R$ is the resistance of $\mathrm{HfO}_{x}, A$ is cross-sectional area of the tip apex (with radius of $75 \mathrm{~nm}$ ) and $L(3.3 \mathrm{~nm})$ is the thickness of the $\mathrm{HfO}_{x}$ protrusion. The transverse resistivity of the $\mathrm{HfO}_{x}$ layer was found to be $4.284 \times 10^{9} \Omega \mathrm{cm}$ which is in agreement with the value reported for sputtered $\mathrm{HfO}_{2}$ films in the literature [25]. When this result is compared with our previous study [24], it can be understood that $\mathrm{HfO}_{x}$ is more resistive than $\mathrm{TaO}_{x}$ (resistivity of $5.76 \times 10^{8} \Omega \mathrm{cm}$ ), as created by the method of AFM local oxidation.

In addition to the $I-V$ characteristics, our sample surface was characterized by different electrical methods available with SPM, such as electric force microscopy (EFM) and spreading surface resistance (SSR). Figures 7 $(a)$ and $(b)$ show the 2D AFM topography and EFM images of a sample with an area of $1.412 \mu \mathrm{m} \times 1.275 \mu \mathrm{m}$ and with a thickness of $3.162 \mathrm{~nm}$. To obtain an EFM image, we used the second pass technique in semi-contact (tapping) mode. In this technique, the surface topography is determined during the first pass in semi-contact operation and then during the second pass, the tip is lifted above the initial position at a constant height $(10 \mathrm{~nm}$ for our study) by applying a dc bias voltage ( $3 \mathrm{~V}$ or our study). Following the trajectory of the tip obtained during the first pass, we obtained the electric field gradient in the $z$-direction on the surface. In figure $7(b)$, darker regions correspond to more electrically resistive regions while brighter 

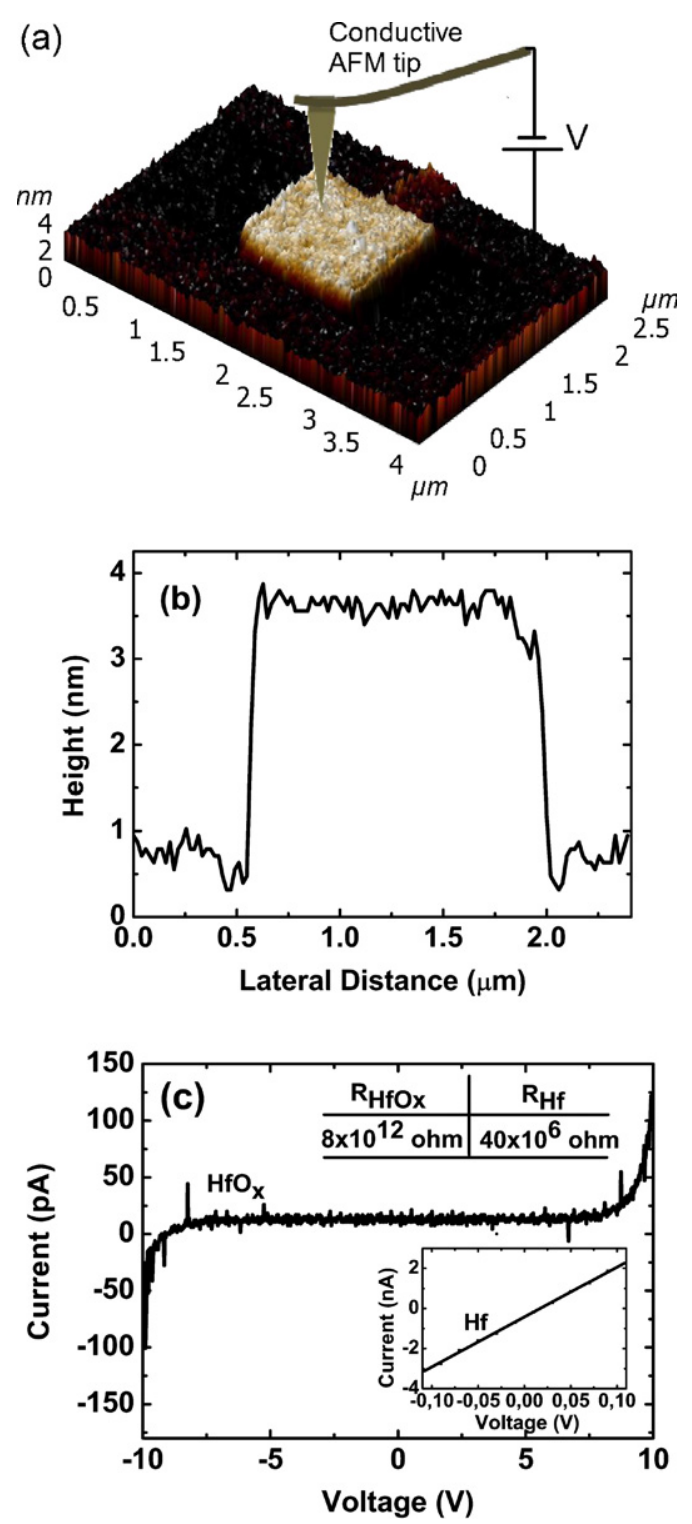

Figure 6. (a) 3D AFM image of $\mathrm{HfO}_{x}$ layer and representation of two terminal electrical measurements. (b) Thickness profile of $\mathrm{HfO}_{x}$ layer. (c) $I-V$ characteristics of $\mathrm{HfO}_{x}$ layer and bare $\mathrm{Hf}$ film (inset).

regions correspond to more conductive regions. Figures 7(a) and $(b)$ prove that an electrochemical surface modification has been obtained by oxidizing locally defined regions on the Hf surface. The topography of oxide protrusions, their corresponding 2D spreading surface resistance (SSR) image (in contact mode) and 1D SR profile are shown in figures 8(a)(c), respectively. In the SR method, a bias voltage is applied to the conductive AFM tip ( $3 \mathrm{~V}$ for our study) and the current passes through the surface during scan, yielding information about the resistance of the surface. For figure $8(b)$, areas of darker contrast represent areas of higher resistance on the surface. This means that the $\mathrm{HfO}_{x}$ surface should be much more resistive than the Hf surface. A difference of $25 \mathrm{pA}$ was found to exist between the surface of the Hf film and the protruded $\mathrm{HfO}_{x}$ surface (figures $8(b)$ and $(c)$ ). In addition to the EFM image (figure 7(b)), the SR image of the
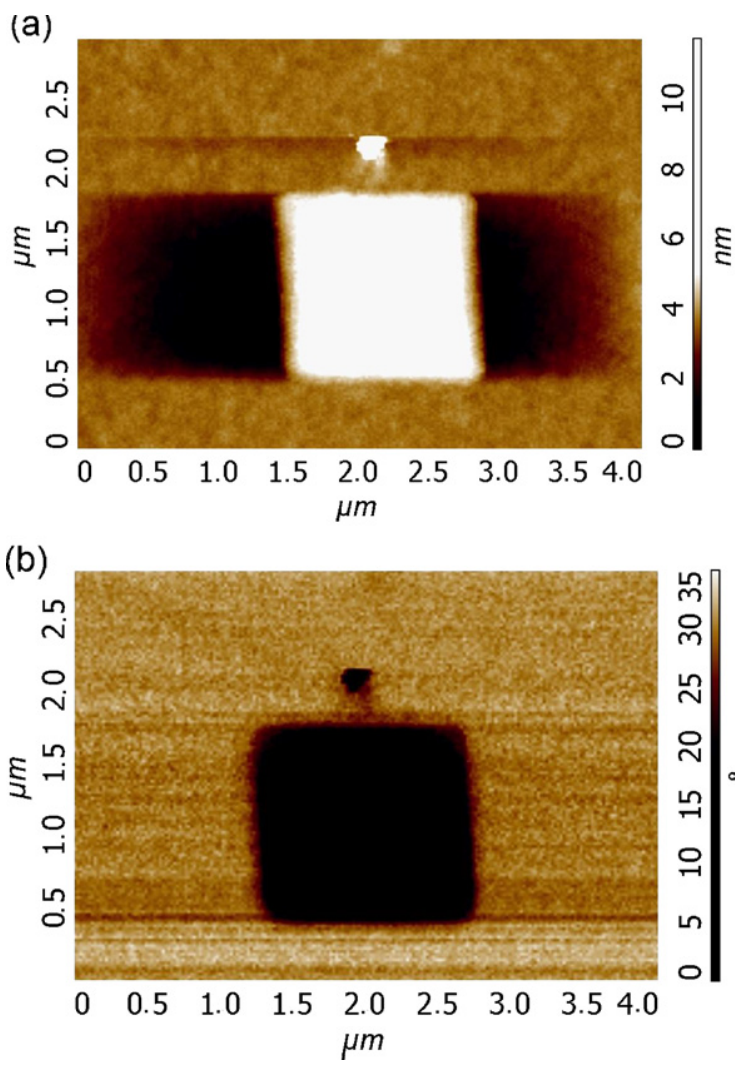

Figure 7. (a) 2D surface topography image and (b) EFM image of Hf surface after local oxidation.

surface also confirms that the surface of Hf was successfully oxidized.

\section{Conclusion}

In summary, oxidation kinetics were investigated for the line patterns of $\mathrm{HfO}_{x}$ dielectric protrusions grown by tip-induced anodic electrochemical oxidation via SC-SPL. The thickness and width of the oxides increase with increasing bias voltage amplitude and duration. However, no oxide patterns were obtained for applied voltages of less than $4.4 \mathrm{~V}$ even at $87 \%$ relative humidity because for low voltages enough kinetic energy is not supplied to the oxyanions to create measurable protrusions on the Hf surface. It was also found that the relative humidity of the environment significantly affects the oxide growth kinetics. The thickness and width of the oxide patterns increase with the increasing relative humidity because of the fact that at high relative humidity there are many more water molecules to provide the oxyanions for the oxidation reaction to proceed. Electrical characterization was also performed on the Hf film and the $\mathrm{HfO}_{x}$ structures directly obtaining $I-V$ curves by means of the conductive AFM tip. Resistivity of the $\mathrm{HfO}_{x}$ structure was found as $4.284 \times 10^{9} \Omega \mathrm{cm}$. It was found that the resistivity of $\mathrm{HfO}_{x}$ is one order of magnitude higher than that of the $\mathrm{TaO}_{x}$ created by the same method. EFM and spreading surface resistance measurements were also successfully performed by implying the resistive material formation on the surface. 

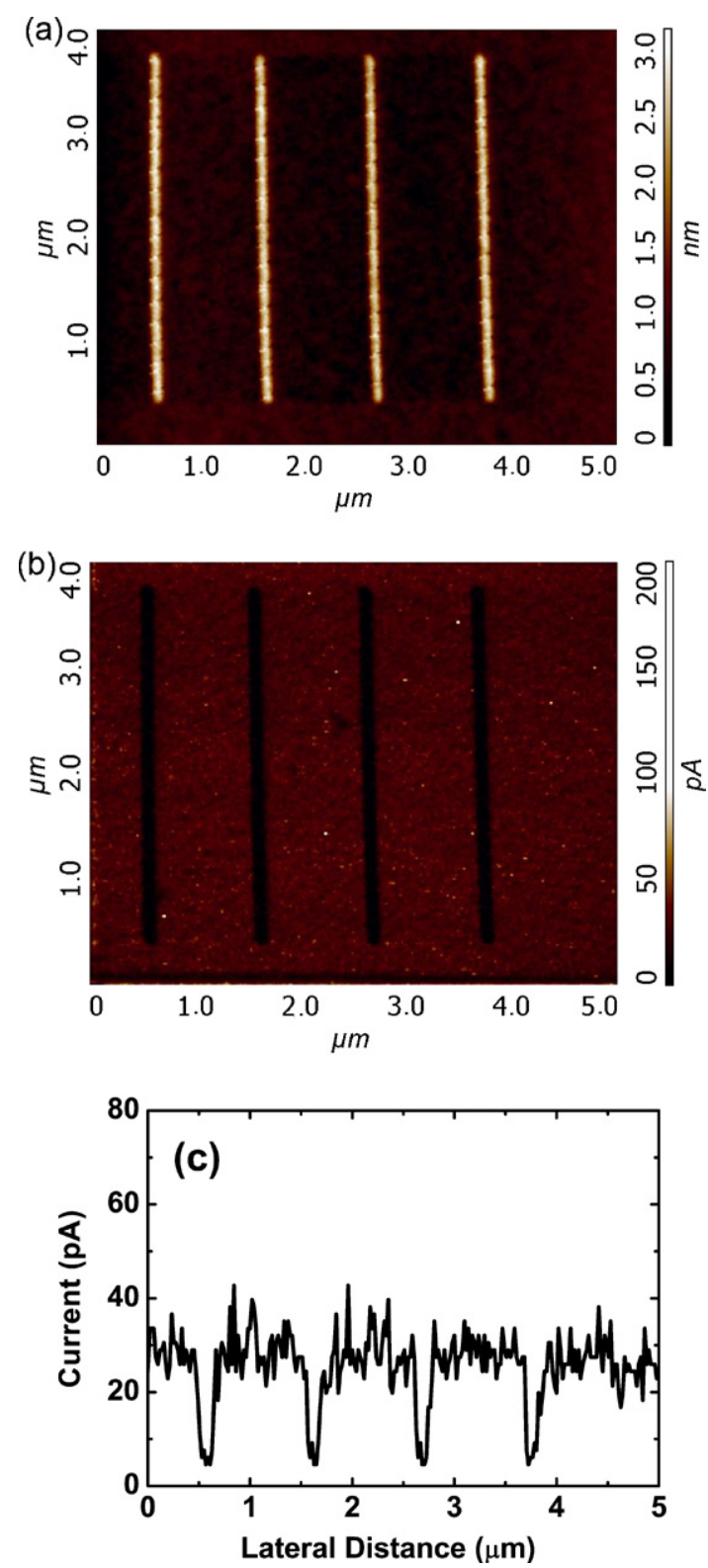

Figure 8. (a) 2D surface topography of $\mathrm{HfO}_{x}$ lines and $(b)$ spreading resistance image and $(c)$ 1D SR current profile of surface.

\section{Acknowledgments}

This research was partially supported by the State Planning Organization of Turkey with the project number of DPT2003K120390, the Izmir Institute of Technology with research project number of 2004IYTE22 and 2006IYTE21 and by the Turkish Research Council (TUBITAK) with project number 107T117. Dr Ritchie Eanes is acknowledged for his technical and grammatical proof reading suggestions.

\section{References}

[1] Suh Y 2003 Fabrication and evaluation of devices containing high $K$ gate dielectrics and metal gate electrodes for the 70 and $50 \mathrm{~nm}$ technology nodes of ITRS PhD Dissertation Department of Electrical Engineering North Caroline State University
[2] Sayan S, Garfunkel E and Suzer S 2002 Soft x-ray photoemission studies of the $\mathrm{HfO} 2 / \mathrm{SiO} 2 / \mathrm{Si}$ system $\mathrm{Appl}$. Phys. Lett. 802135

[3] Manchanda L, Morris M D, Green M L, van Dover R W, Klemens F, Sorsch T W, Silverman P J, Wilk G, Busch B and Aravamudhan S 2001 Multi-component high-K gate dielectrics for the silicon industry Microelectron. Eng. 59 351-9

[4] Kang L, Hun Lee B, Qi W-J, Jeon Y, Nieh R, Gopalan S, Onishi K and Lee J C 2000 Electrical characteristics of highly reliable ultrathin hafniumoxide gate dielectric Electron Dev. Lett. 21 181-3

[5] Hun Lee B, Kang L, Qi W-J, Nieh R, Jeon Y and Onishi Lee K 1999 MOSCAP and MOSFET characteristics using $\mathrm{ZrO}_{2}$ gate dielectric deposited directly on Si IEDM Tech.

[6] Chaneliere C, Autran J L, Devine R A B and Balland B 1998 Tantalum pentoxide $\left(\mathrm{Ta}_{2} \mathrm{O}_{5}\right)$ thin films for advanced dielectric applications Mater. Sci. Eng. 22 269-322

[7] Rai-Chaudhury P 1997 Handbook of Microlithography, Micromachining, and Microfabrication (Bellingham, WA: SPIE)

[8] Gotoh Y et al 2000 Experimental and theoretical results of room-temperature single-electron transistor formed by the atomic force microscope nano-oxidation process J. Vac. Sci. Technol. A 18 1321-5

[9] Lee J-H, Miyata N, Kundu M and Ichikawa M 2002 Oxidation

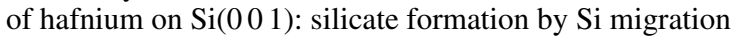
Phys. Rev. B 66233309

[10] Aygun G, Atanassova E, Alacakir A, Ozyuzer L and Turan R 2004 Oxidation of Si surface by a pulsed Nd : YAG laser $J$. Phys. D: Appl. Phys. 37 1569-75

[11] Atanassova E, Aygun G, Turan R and Babeva Tz 2006 Structural and optical characteristics of tantalum oxide grown by pulsed Nd: YAG laser oxidation J. Vac. Sci. Technol. 24 206-11

[12] Lo R L, Lee W C and Kwo J 2006 AFM nano-lithography on hafnium oxide thin film grown on $\operatorname{Si}\left(\begin{array}{l}1 \\ 0\end{array}\right)$ Japan. J. Appl. Phys. 45 2067-9

[13] Soh H T, Guarini K W and Quate C 2001 F Scanning Probe Lithography (Boston, MA: Kluwer)

[14] G Ricardo, Martinez R V and Martinez J 2006 Nano-chemistry and scanning probe nanolithographies Chem. Soc. Rev. 35 29-38

[15] Garcia R, Calleja M and Rohrer H 1999 Patterning of silicon surfaces with noncontact atomic force microscopy: field-induced formation of nanometer-size water bridges $J$. Appl. Phys. 861898

[16] Irmer B, Kehrle M and Kotthaus J P 1997 Fabrication of $\mathrm{Ti} / \mathrm{TiO}_{x}$ tunneling barriers by tapping mode atomic force microscopy induced local oxidation Appl. Phys. Lett. 711733

[17] Teuschler T, Mahr K, Miyazaki S, Hundhausen M and Ley L 1995 Nanometer-scale field-induced oxidation of

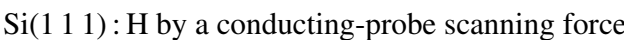
microscope: doping dependence and kinetics Appl. Phys. Lett. 673144

[18] Dagata J A, Perez-Murano F, Abadal G, Morimoto K, Inoue T, Itoh J and Yokoyama H 2000 Predictive model for scanned probe oxidation kinetics Appl. Phys. Lett. 762710

[19] Avouris Ph, Hertel T and Martel R 1997 Atomic force microscope tip-induced local oxidation of silicon: kinetics, mechanism, and nanofabrication Appl. Phys. Lett. 71285

[20] Ma Y R, Yu C, Yao Y D, Liou Y and Lee S F 2001 Tip-induced local anodic oxidation on the native $\mathrm{SiO} 2$ layer of Si(1 11 1) using an atomic force microscope Phys. Rev. B 64195324

[21] Lu Y S, Wu H I, Wu S Y and Ma Y R 2007 Tip-induced large-area oxide bumps and composition stoichiometry test via atomic force microscopy Surface Sci. 601 3788-791 
[22] Fang T-H and Chang W-J 2004 Nanolithography and nanoindentation of tantalum-oxide nanowires and nanodots using scanning probe microscopy Physica B 352 190-9

[23] Farkas N, Tokash J C, Zhang G, Evans E A, Ramsier R D and Dagata J A 2004 Local oxidation of metal and metal nitride films J. Vac. Sci. Technol. A 22 1879-84
[24] Okur S, Büyükköse S and Tari S 2008 Scanning probe oxidation on Ta thin films $J$. Nanosci. Nanotechnol. 8 5640-5

[25] Huber F 1971 Properties of hafnium dioxide thin-film capacitors IEEE Trans. Parts, Hybrids Packag. 7 141-7 